\title{
Vibrational and acoustic characteristics of soundboard (Acoustical research on the piano, Part 3)
}

\author{
Isao Nakamura \\ Teikyo University of Technology, \\ 2289 Uruido, Ichihara, Chiba, 290-01 Japan
}

(Received 31 March 1993)

【English translation of the same article in J. Acoust. Soc. Jpn. (J) 37, 510-517 (1981)】

\begin{abstract}
The soundboard of a piano without strings vibrates in almost the same way as in an isotropic plate in a low-frequency region, because the anisotropy of its wooden material is compensated by its ribs, while the soundboard vibrates more locally in a high-frequency region. The boundary condition of the soundboard in a low-frequency region is a mixture of the fixed and simply supported conditions, whereas it becomes a fixed-edge state in a high-frequency region. Wher strings are added to the soundboard, they increase the resonance frequency of each vibration mode on the soundboard, and reduce its $Q$ value. Characteristics of a soundboard can be quantitatively shown by measuring an admittance at the driving point of each string on the bridge. The admittance measured is also important to indicate the degree of influence of strings on the soundboard. The soundboard vibrates in a low-frequency range, reducing the amplitude of the fundamental vibration, and emphasizing its partials since the driving points are near the edge. In an intermediate-frequency region, the fundamental mode contributes very little to vibrations since it is low than the frequency range. The admittance in a high-frequency region is high due to local vibrations occurring between ribs. Other acoustic characteristics of the soundboard, such as its vibrations with a constant driving force, and the sound-pressure directivity of soundboards, have been investigated.
\end{abstract}

Keywords: Soundboard without strings, Soundboard with strings, Eoundary condition, Driving point admittance, Acoustic characteristics

PACS number: 43. 75. Wn, 43. 40. Dx, 43. 40. $\mathrm{Rj}$

\section{INTRODUCTION}

The soundboard of a piano is a component to form a piano sound from its strings, and to radiate it into the air. The energy given to a string by striking it with a hammer pulsively is radiated from the soundboard taking a relatively long time. The soundboard can be regarded as a sound source which has a wide area and a wide frequency region.

Each piano maker has standardised the principal structures of its soundboards in the last few decades. Although there have been many new ideas published in patents in various countries including Japan, almost none of them has been used for practical prod- ucts. It can be said, therefore, that the structure of soundboards has now been completed as far as wood is used. On the other hand, there have been very few published papers ${ }^{1)}$ on the characteristics of piano soundboards: a paper on an improvement of the soundboard of a grand piano by P. H. Bilhuber and C. A. Johnson (1940), ${ }^{2)}$ other papers by Nakamura et $a l^{3,4}$ )

This paper aims at clarifying the characteristics of the soundboard of an upright piano by measuring its various vibration and acoustic characteristics. Firstly, the vibration modes of a soundboard without strings is studied, then those with strings are examined with results of measurements. It has been 
found that the frequency characteristics of the driving point admittance at the joint of strings and the soundboard have a very significant contribution to the formation of timbre of a piano and its acoustic characteristics. Therefore, the driving point characteristics and the acoustic characteristics of representative keys are investigated. Finally, the directivity of a piano in a sound field is described.

\section{VIBRATION MODES}

Figure 1(a) shows an example of the soundboard of an upright piano in a middle price range. The soundboard usually consists of more than ten pieces of straight-grained wood (e.g. spruce) glued together to form a single plane, and other square timbers ("sound ribs," the height and width being around $20 \mathrm{~mm}$ depending on the position on the soundboard) are glued on the plane across its grain. The thickness of the sound ribs is gradually thinner towards their edges, and the edges of the soundboard are fixed to an iron frame through a wooden frame. On the soundboard opposite to these sound ribs, two types of bridge ('main bridge' and 'bass bridge,' both made of square timber of maple or beech) are glued to connect the soundboard and strings. The bass bridge used for lower notes consists of two pieces with a triangle plane between them.

Figure 1(b) shows a soundboard specially made for the experiment, and this has exact rectangular edges without the trimming rims. The thickness of the main part of the experimental soundboard is $8.5 \mathrm{~mm}$, and this is slightly thinner in a higher tone range. The dotted lines in Fig. 1(b) show the positions of the sound ribs on the other side of the board.

Figure 2 shows Chladni figures of the soundboard at various natural frequencies driven at the centre without strings, Fig. 2(a) being a conventional soundboard, and Fig. 2(b) the experimental soundboard. The former shows that the vibration modes are modified by its trimming ribs. The latter, which uses a rectangular board, shows that the vibration modes at lower frequencies are almost isotropic; notably at the frequency lower than 600 $\mathrm{Hz}$, the modes are not affected by the main bridge and the sound ribs. At the frequencies higher than $300 \mathrm{~Hz}$, however, the vibration modes of the $y$ direction (see Fig. 1) in a higher-note region (the right side) tend to form more groups than those in

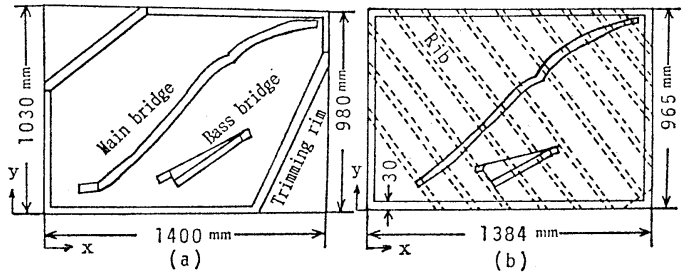

Fig. 1 (a) Typical soundboard of an upright piano. (b) Experimentally made rectangular soundboard without trimming rims.
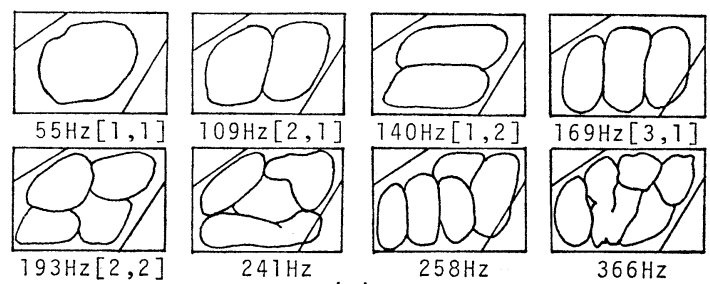

(a)
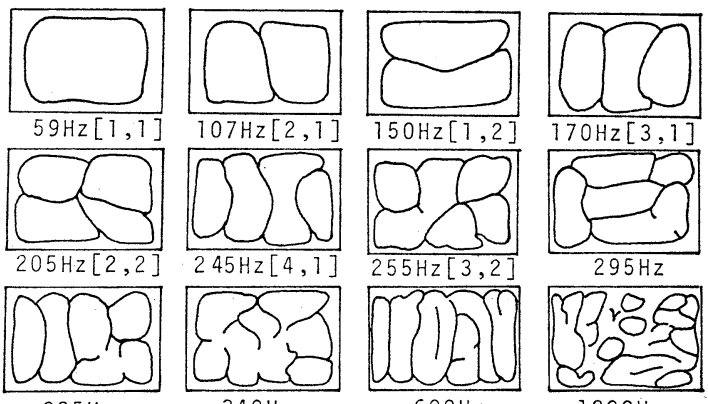

$349 \mathrm{~Hz}$

(b)
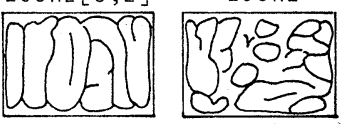

$1200 \mathrm{~Hz}$

Fig. 2 Chladni figures of soundboards without strings. (a) Typical soundboard. (b) Experimental soundboard.

the lower-note region (the left side). This is due to the thickness of the soundboard. The Chladni figure for $1,200 \mathrm{~Hz}$ clearly shows the influence of the main bridge. At the frequencies higher than this (not illustrated), vibrations between sound ribs can be seen.

Assuming that the anisotropy of the woods used for the soundboard is compensated by its sound ribs, and that the main bridge can transmit a force uniformly to discontinuously arranged sound ribs (i.e. the soundboard is an isotropic plate), the frequency of the $m n$th mode, $f_{m n}$, is given by 


\section{NAKAMURA: CHARACTERISTICS OF SOUNDBOARD}

$$
f_{m n}=\frac{d}{2 \pi} \sqrt{\frac{E}{12 \rho\left(1-\mu^{2}\right)}}\left(\frac{\lambda_{m}{ }^{2}}{l_{x}{ }^{2}}+\frac{\lambda_{n}{ }^{2}}{l_{y}{ }^{2}}\right)
$$

where $m$ and $n$ are the mode numbers in the $x$ and $y$ directions respectively, $\lambda_{m}$ and $\lambda_{n}$ are the eigen values, $l_{x}$ and $l_{y}$ are the lengths of the soundboard in the $x$ and $y$ directions respectively, $d$ is the equivalent thickness of the soundboard, $\rho$ is the density, $E$ is the Young's modulus, and $\mu$ is the Poisson ratio. The eigen values are determined by the boundary conditions; $\pi, 2 \pi, 3 \pi$ for a simply supported boundary, and

$$
\cos \lambda_{m} \cosh \lambda_{m}=1, \quad \cos \lambda_{n} \cosh \lambda_{n}=1
$$

for a fixed boundary, where $\lambda_{1}=4.730, \lambda_{2}=7.853$, $\lambda_{3}=10.996, \ldots$.

Figure 3 shows resonance frequencies of the soundboards with three boundary conditions and three different orders of mode numbers. The resonance frequencies are normalized to the respective fundamental resonance frequencies of the $[1,1]$ mode. Chain lines show theoretical values for the simply supported boundary; dotted lines show theoretical values for the fixed boundary; and solid lines show measured values from the experimental soundboard shown in Fig. 1(b). In Figs. 3 (a) and (b), the values are arranged in the order of $x$-mode numbers, with the $y$-mode number fixed to 1, and 2, respectively. In Fig. 3(c), the values are arranged in the order of $y$-mode numbers, with the $x$-mode number fixed to 1 . In this arrangement, Chladni figure for $295 \mathrm{~Hz}$ shown in Fig. 2(b) is regarded as a variation of [1,3] mode. The measured values lie between those calculated for simply supported and fixed boundaries. Comparing the gradients of the curves in Fig. 3, the experimental soundboard is similar to a soundboard with a simply supported boundary at a low frequency range, but this is similar to a soundboard with a fixed boundary at a high frequency range.

Referring to Figs. 2(b) and 3, the appearance of two modes $[4,1]$ and $[3,2]$ can be summarized as follows: -

$\begin{array}{lcc} & \begin{array}{c}\text { Low } \\ \text { frequency } \\ \text { side }\end{array} & \begin{array}{c}\text { High } \\ \text { frequency } \\ \text { side }\end{array} \\ \text { Fixed soundboard } & {[4,1]} & {[3,2]} \\ \begin{array}{c}\text { Simply supported } \\ \text { soundboard }\end{array} & {[3,2]} & {[4,1]} \\ \begin{array}{c}\text { Experimental } \\ \text { soundboard }\end{array} & {[4,1]} & {[3,2]}\end{array}$

Note, the two modes are reversed in the top and second lines. The ratio of the frequency in $[1,1]$ modes with the fixed soundboard to that of the simply supported soundboard is 2.27 . This suggests that the low frequency modes are influenced by the boundary condition of the soundboard.

The acoustic load is neglected in these calculations, and this is equivalent to reducing the fundamental frequency by about $5 \mathrm{~Hz}$. This neglect has little effect on the relative values of the boundary conditions.

Figure 4 shows contour lines of vibrations of the

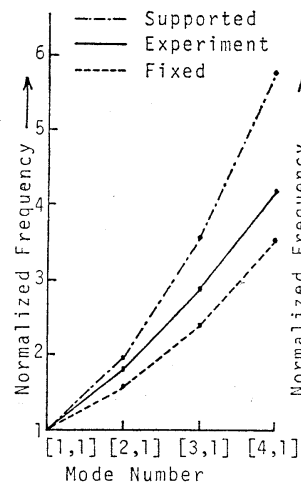

(a)

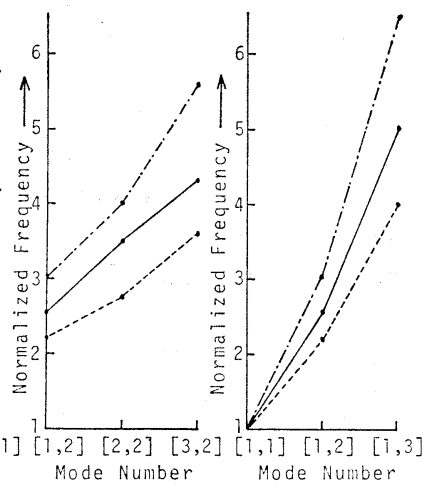

(c)

Fig. 3 Normalized frequencies of a rectangular soundboard (calculated and measured) arranged in different orders of node numbers.

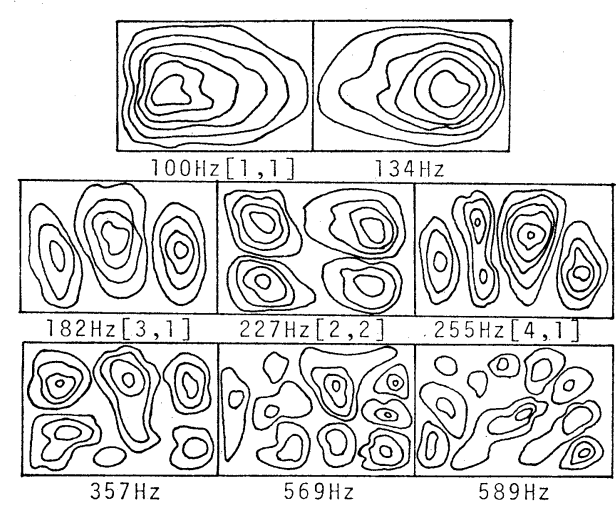

Fig. 4 Contour lines of vibrations of a rectangular soundboard with strings. A sinusoidal force was applied to the centre of the soundboard. 
experimental soundboard (rectangular) with strings. Given a driving force to the centre of the soundboard, the distribution of sound pressures on its surface was measured. The curves in the figures can be regarded as iso-amplitude lines: intervals of the lines being arbitrary linear units. To measure the sound pressure near the surface of the board has advantages in that the measurements not only detect the positions of nodes but also local vibrations, although this method is affected by the directivity of a microphone used. The driving force given to each of the cases in Fig. 4 is not uniform throughout; a lower input force being used when the centre of the soundboard has a loop, and a higher input force when that has a node. The $[1,1]$ mode appears at $100 \mathrm{~Hz}$ (driven by a small force) and $134 \mathrm{~Hz}$ (driven by a large force). At each frequency, the maximum amplitude occurs at a position other than the centre of the soundboard. This is due to the main bridge. The pattern with $134 \mathrm{~Hz}$ is considered to be a pseudo mode because of the large driving force. Adding strings to the soundboard remains the patterns of modes unchanged, but the sound pressure level reduces, and the resonant frequencies increase. This tendency is more clearly seen in the low frequency range.

\section{EFFETS OF STRINGS}

Let us theoretically examine the increase of the resonant frequency of the soundboard and the reduction of its $Q$ value due to adding strings to it. Let us assume that a string is set on the soundboard through a bridge as shown in Fig. 5. The string is divided by the bridge into two parts, short and long, and the hammer strikes the longer part. The angles $\theta_{1}$ and $\theta_{2}$ are formed by the string and the surface of the soundboard as shown in Fig. 5, the former being greater than the latter. The soundboard is usually made with a lightly curved surface so that this becomes flatter when strings are set. Although this produces a distributed shear stress inside the soundboard, this is neglected in this discussion, and the effects of strings on the vibrations alone are analysed. When the hammer does not strike the string, the longer side of the string can be regarded as characteristic impedance, since this is terminated by felt of the damper so that almost no reflection occurs in the string. The shorter side of the string acts as a stiffness in the vibration of the bridge. The driving-point impedance of the $n$-th

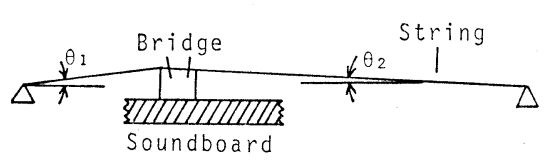

Fig. 5 Geometrical relationship between soundboard, bridge and string.

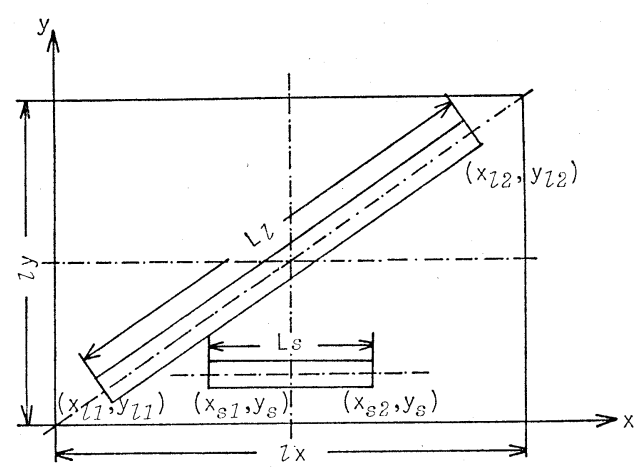

Fig. 6 Simplified soundboard for calculations.

string $Z_{n}$ is given by

$$
Z_{n} \fallingdotseq \sqrt{\sigma_{n} T_{n}}+\frac{1}{j \omega a_{n} / T_{n}}
$$

where $T_{n}$ is the tension of the string, $\sigma_{n}$ is the line density, $a_{n}$ is the length of the shorter part of the string. Note, $Z_{n}$ should be two and three times for double and triple strings respectively. The load impedances for each of key numbers 1 to 88 are given by

$$
Z=\sum_{n=1}^{88} \sqrt{\sigma_{n} T_{n}}+\frac{1}{j \omega} \sum_{n=1}^{88} \frac{T_{n}}{a_{n}} .
$$

Since this is based on an assumption that all strings vibrate in-phase to each other; i.e., the vibration of the soundboard is a piston motion with $[0,0]$ mode, some corrections are required in their results depending on the shape of each mode. Assuming that the soundboard has a simply supported boundary condition (for simplicity in a calculation), the normalized functions in the $x$ and $y$ directions are $\sin m \pi x / l_{x}, \sin n \pi y / l_{y}$. Let us now calculate the ratio $A_{\mathrm{s}}$ of the stiffness acting on the soundboard with strings and the stiffness of the soundboard which is assumed to be vibrating in a piston motion with $[0,0]$ mode. To simplify the calculation, it is asumed that the soundboard has a structure as shown in Fig. 6; i.e. the main bridge is on the 


\section{NAKAMURA: CHARACTERISTICS OF SOUNDBOARD}

diagonal of the soundboard, and the bass bridge is parallel to the $x$ axis (an actual intermediate plate is ignored). The $A_{\mathrm{s}}$ is given by

$$
A_{\mathrm{s}}=\frac{1}{L_{1}+L_{\mathrm{s}}} \int_{L_{1}}\left|\sin m \pi x / l_{x} \cdot \sin n \pi y / l_{y}\right| d L_{1}
$$

$$
A_{\mathrm{s}}=\frac{\sqrt{1+\left(l_{y} / l_{x}\right)^{2}}}{L_{1}+L_{\mathrm{s}}} \int_{x l_{1} \sqrt{1+\left(l_{y} / l_{x}\right)^{2}}}^{x_{l_{2}} \sqrt{1+\left(l_{y} / l_{x}\right)^{2}}}\left|\sin m \pi x / l_{x} \cdot \sin n \pi x / l_{x}\right| d x+\frac{1}{L_{1}+L_{\mathrm{s}}}\left|\sin n \pi y_{*} / l_{y}\right| \int_{x_{\mathrm{s}_{1}}}^{x_{\mathrm{s}}}\left|\sin m \pi x / l_{x}\right| d x
$$

Using constants for the soundboard: $l_{x}=132.4, l_{y}=$ 90.5,

$$
\begin{array}{ll}
\left(x_{1_{1}}, y_{1_{1}}\right)=(22.0,15.0), & \left(x_{1_{1}}, y_{1_{1}}\right)=(102.4,89.5), \\
\left(x_{\mathrm{s}_{1}}, y_{\mathrm{s}}\right)=(52.5,20), & \left(x_{\mathrm{s}_{2}}, y_{\mathrm{s}}\right)=(92.0,20),
\end{array}
$$

$A_{\mathrm{s}}$ for $[1,1]$ mode is given by

$$
A_{\text {s }}=0.293
$$

From Eq. (4), the resistance due to the strings is

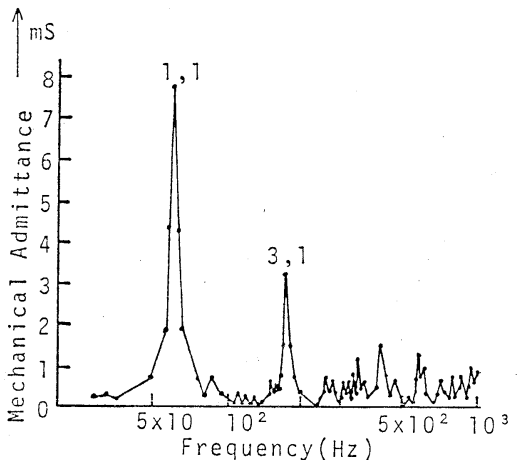

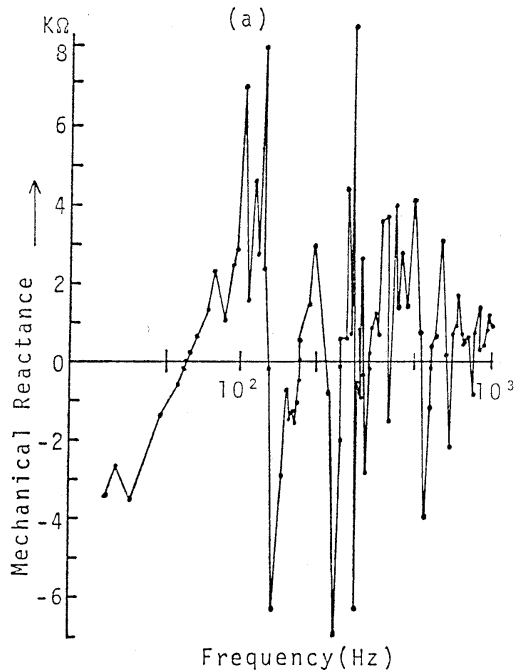

(a')
$474 \Omega$, and the stiffness due to the strings is $2.42 \times$ $10^{6} \mathrm{~N} / \mathrm{m}$. Therefore, the additional resistance caused by the $[1,1]$ vibration is $139 \Omega$, and that of the stiffness is $0.71 \times 10^{6} \mathrm{~N} / \mathrm{m}$.

\section{CHARACTERISTICS OF DRIVING POINT}

The impedance of the soundboard at the bridge

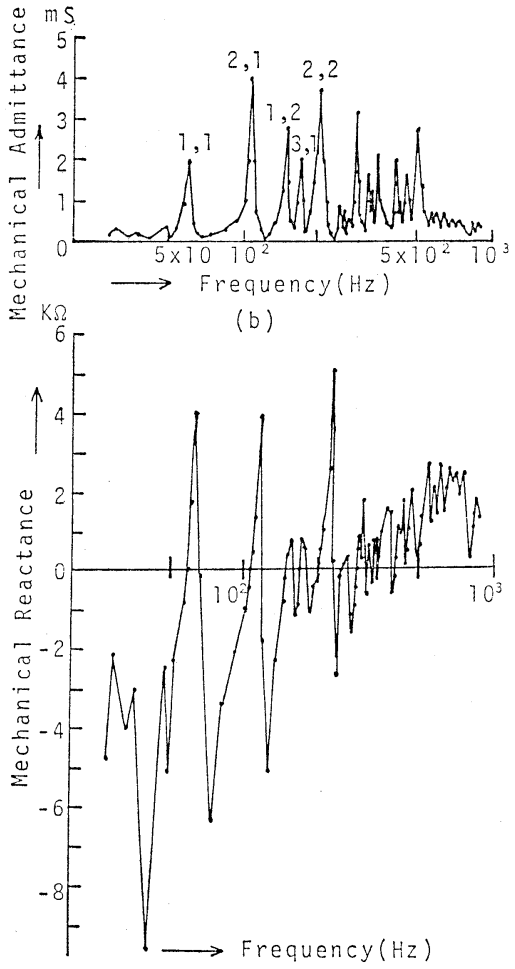

$\left(b^{\prime}\right)$

Fig. 7 Frequency characteristics of mechanical admittance and reactance (at the driving point) of a rectangular soundboard without strings measured at main bridge. Driving point: - (a) and $\left(a^{\prime}\right)$ : Key No. 48; (b) and $\left(b^{\prime}\right)$ : Key No. 30. 
which connects a string and the soundboard is an important element not only as a load of the vibration of the string, but as an indicator of the characteristic of the soundboard. The driving point admittance is obtained by driving the soundboard with a constant current driver (an apparatus modified from a high-powered dynamic-type loudspeaker), and by measuring the vibration velocity at this driving point. The phase difference between the driving force and the vibration velocity is also measured with a calibrated phase-detector. Then the reactance and the resistance components can be calculated from the admittance and the phase difference.

Figure 7 shows a typical driving-point admittance and reactance of the soundboard at the main bridge without strings. (a) shows the results ob- tained by driving the soundboard at the position of key No. 48 (near the centre of the soundboard), and (b) shows that at key No. 30 (near the edge).

Figure 8 shows similar results to Fig. 7 but at the bass bridge. (a) shows the results obtained by driving the soundboard at the position of key No. 1 (near the left edge of the soundboard), (b) at the position of key No. 15 (near the centre of the soundboard), and (c) at the position of key No. 25 (near the right edge of the soundboard).

Let us examine the driving characteristics on the main bridge. When the centre of the soundboard is driven, the admittances in odd-number modes such as $[1,1]$ and $[3,1]$ are large since they form resonance points, and the admittance in even-number modes are small since they form nodes. When the edge of the soundboard is driven, all the modes

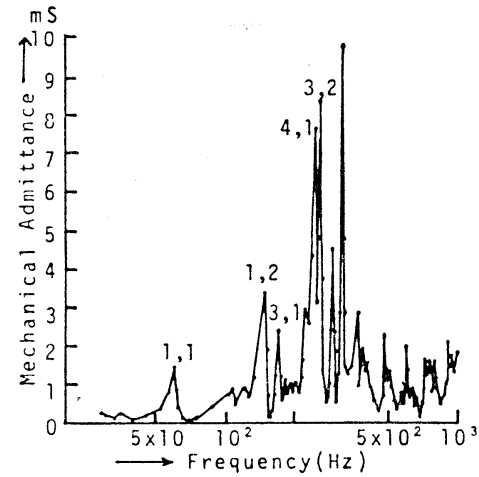

(a)

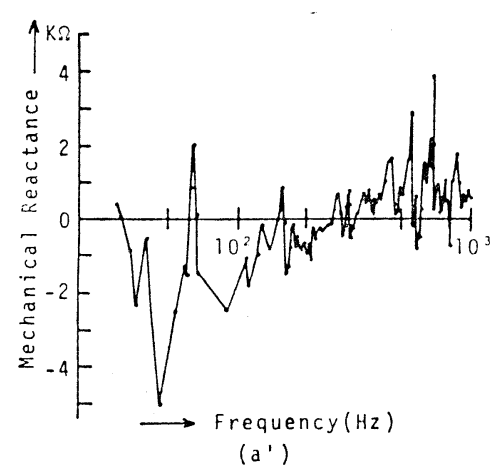

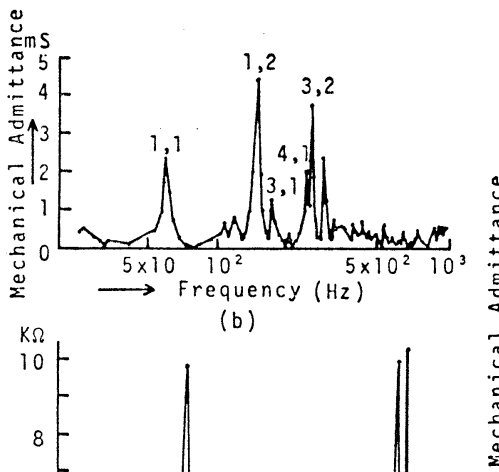

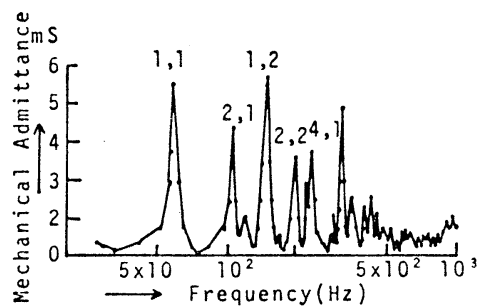

(c)

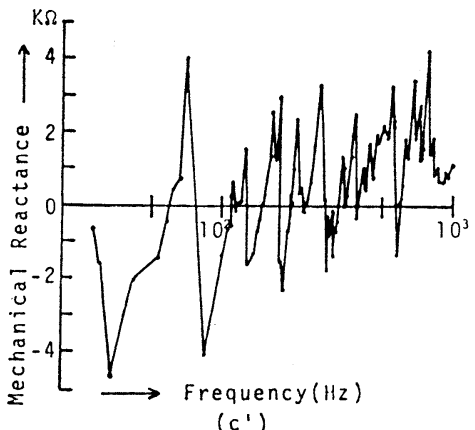

(c')

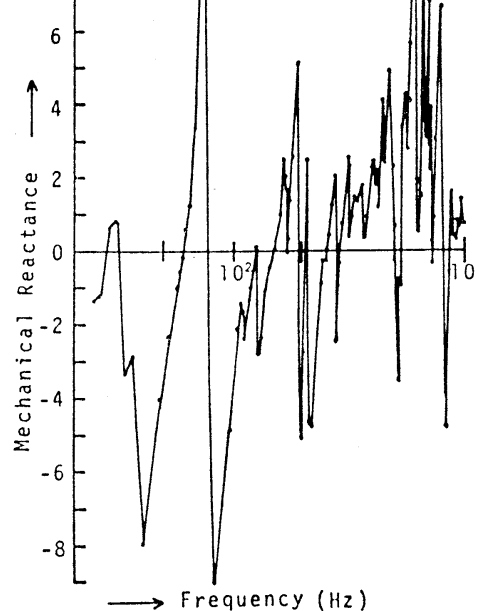

(b')

Fig. 8 Frequency characteristics of mechanical admittance and reactance (at the driving point) of a rectangular soundboard without strings measured at bass bridge. Driving point:-(a) and $\left(a^{\prime}\right)$ : Key No. 1 ; (b) and $\left(b^{\prime}\right)$ : Key No. $1.5 ;(c)$ and $\left(c^{\prime}\right)$ : Key No. 25. 


\section{NAKAMURA: CHARACTERISTICS OF SOUNDBOARD}

appear as resonance points, although the admittance in $[1,1]$ mode becomes small. Referring to the reactance characteristics, the reactance varies depending on each mode. When the soundboard is driven at its centre, the frequency-reactance characteristic varies around zero. When the soundboard is driven at its edge, it appears as if a LC system having a resonant frequency slightly higher than 200 $\mathrm{Hz}$ is inserted in series. Referring to the driving characteristics on the bass bridge, the admittance at a lower frequency increases when the driving point is moved toward the right edge. When the driving point moves toward the left, the lower-frequency mode is suppressed, and the middle-frequency mode increases. The reactance has a maximum at the centre of the bass bridge, and this reduces at its two edges. At the bass bridge, it appears that a LCsystem having a resonant frequency slightly higher than $200 \mathrm{~Hz}$ is inserted.

Figures 9 and 10 show the driving-point characteristics with strings. Figure 9 corresponds to Fig. 7, and Fig. 10 to Fig. 8 respectively. It can be seen that the resonance point in the characteristic with strings shifts toward a higher frequency, and the $Q$ reduces compared with those without strings. This trend is more conspicuous in a low frequency region.

In the mode $[1,1]$ when driver at the center, the resonance frequency $f_{1}$, effective mass $M_{1}$, effective compliance $K_{1}$ are obtained from the reactance curves: -

Without strings

$f_{1 o}=59 \mathrm{Hy}, \quad M_{1 o}=3.4 \mathrm{~kg}, \bar{K}_{10}=2.14 \times 10^{-6} \mathrm{~m} / \mathrm{N}$.

With strings

$f_{1 \mathrm{w}}=100 \mathrm{~Hz}, M_{1 \mathrm{w}}=3.4 \mathrm{~kg}, \bar{K}_{1 \mathrm{w}}=7.45 \times 10^{-7} \mathrm{~m} / \mathrm{N}$.

Therefore, the compliance and stiffness due to the strings are

$$
\bar{K}_{\mathrm{s}}=1.14 \times 10^{-6} \mathrm{~m} / \mathrm{N}, \quad \text { and } S_{\mathrm{s}}=0.88 \times 10^{-7} \mathrm{~N} / \mathrm{m}
$$

respectively. These almost agree with the theoretical values obtained previously. The differences between the theoretical and measured values are caused by:-(1) The vibrations on the board are assumed to be perfectly sinusoidal; (2) the structure of the brings are simplified (to simplify the calculation); (3) the stiffness due to the strings is assumed to be distributed uniformly; and (4) variations of the effective mass due to those of the boundary conditions are not taken into account.

Figure 11 shows the frequency characteristics of
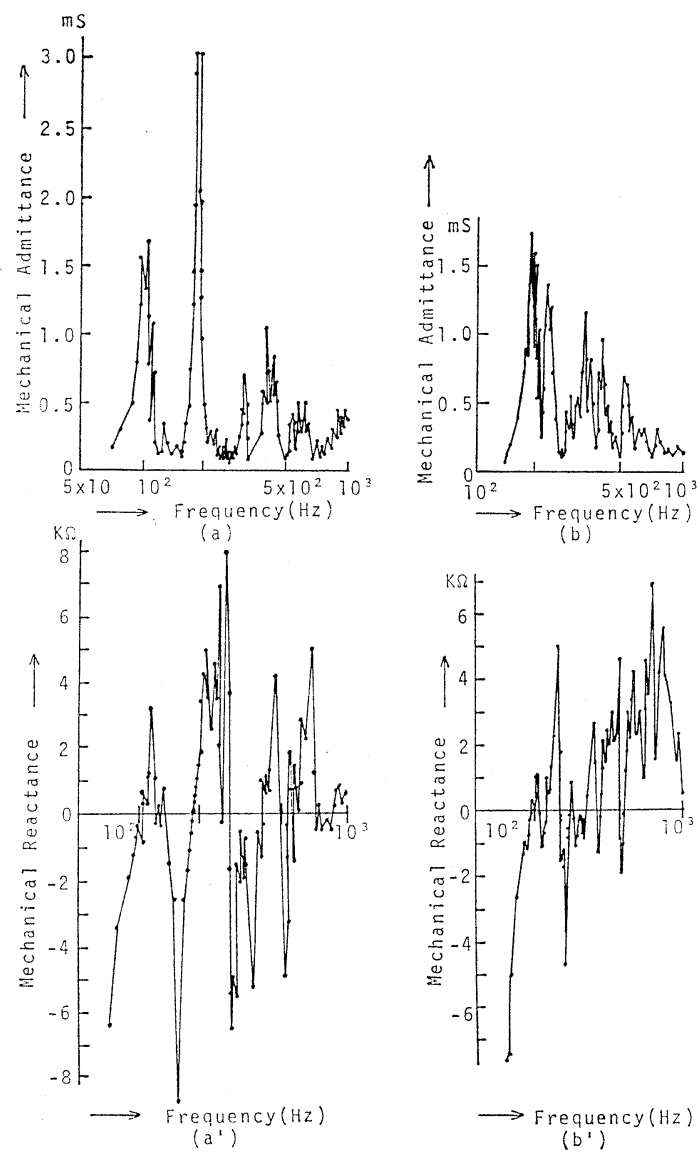

Fig. 9 Frequency characteristics of mechanical admittance and reactance (at the driving point) of a rectangular soundboard with strings measured at main bridge. Driving point:-(a) and $\left(\mathrm{a}^{\prime}\right)$ : Key No. $48(415.305 \mathrm{~Hz}) ;\left(\right.$ b) and $\left(b^{\prime}\right)$ : Key No. 30 (146.832 Hz).

the velocity amplitude of a practical soundboard with strings driven by a constant driving force; the driving point being (a) near the left edge of the bass bridge, (b) near its right edge, (c) the nearest edge of the main bridge, and (d) near its centre. Arrows show resonance frequencies of the driving instrument, and double arrows show the resonance frequencies of the detecting instrument. The frequency curves below $1 \mathrm{kHz}$ show the driving point admittance. The frequency curves above $1 \mathrm{kHz}$ suggest that there are resonance points above this frequency, although these curves are influenced by the measuring instrument. The vibrations below $50 \mathrm{~Hz}$ seems 

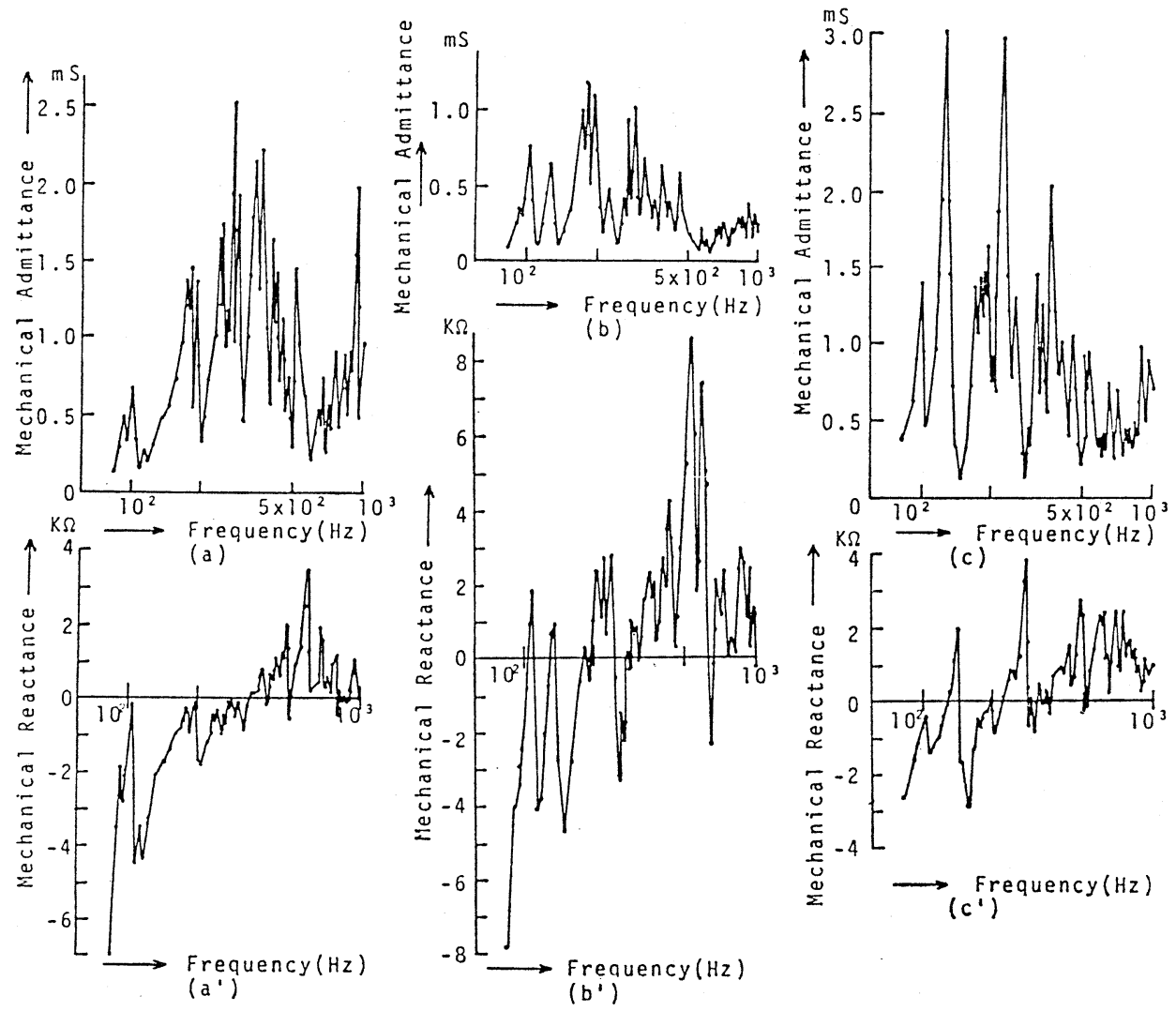

Fig. 10 Frequency characteristics of mechanical admittance and reactance (at the driving point) of a rectangular soundboard with strings measured at bass bridge. Driving point: - (a) and $\left(a^{\prime}\right)$ : Key No. $1(27.5 \mathrm{~Hz})$; (b) and $\left(b^{\prime}\right)$ : Key No. $15(61.735 \mathrm{~Hz})$; (c) and $\left(c^{\prime}\right)$ : Key No. $25(110 \mathrm{~Hz})$.

to be due to a piston motion with $[0,0]$ mode. The fact that the driving-point admittance with an edge driving is rather high at frequencies above $1 \mathrm{kHz}$ is due to the vibration between sound ribs. There is a tendency for the vibrations between the sound ribs in a higher frequency to be divided into many sections in the direction of wooden-board grain.

The reasons why a soundboard has a wide range of frequency characteristic is that the board is isotropic at a low frequency due to the effect of sound ribs, and that a high-frequency characteristic is obtained by the vibrations of internal board to which the sound ribs work as fixed edges.

It appears that the $Q$ of a soundboard is effectively adjusted to an appropriate value by the characteristic impedance of strings.
It is effective to adjust the driving-point admittance appropriately for obtaining a good piano sound with a suitable damping rate.

\section{ACOUSTIC CHARACTERISTICS}

Figure 12 shows the frequency characteristics of sound pressure levels around driving points on a typical soundboard. Each key number corresponds to that in Fig. 11. These records are obtained by an automatic measuring instrument, and show the sound pressure level having frequencies of $80 \mathrm{~Hz}$ to $4 \mathrm{kHz}$. The records of a frequency range higher than $1 \mathrm{kHz}$ are influenced by the resonance characteristic of the driving instrument, notably those higher than $4 \mathrm{kHz}$ which are hardly recorded. The actual sounds are, however, generated up to a much higher frequency. 


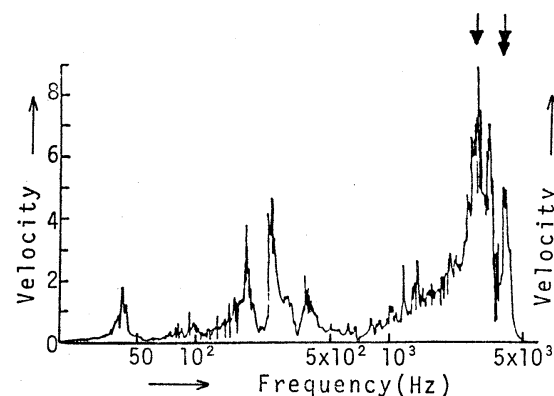

(a)

(c)

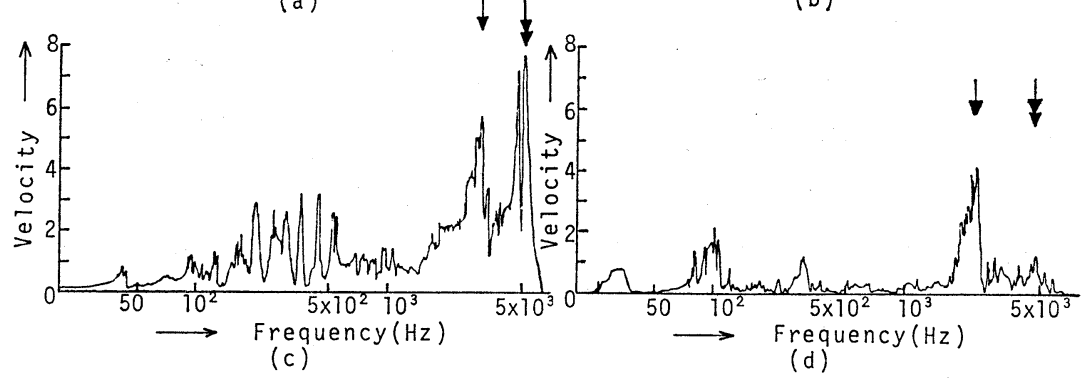

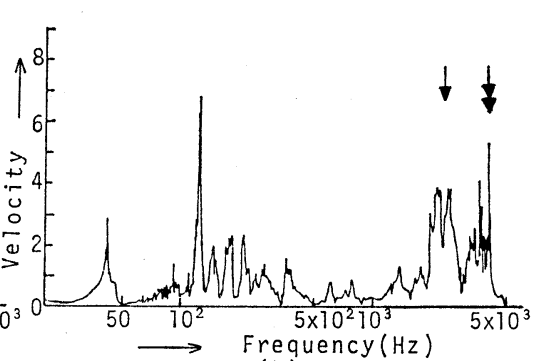

(b)

Fig. 11 Frequency characteristic of velocity-amplitude of a typical sound board with strings, driven by a constant force. Driving point and frequency: - (a) Key No. 1 $(27.5 \mathrm{~Hz})$; (b) Key No. $25(110 \mathrm{~Hz})$; (c) Key No. $27(123.47 \mathrm{~Hz})$; (d) Key No. 40 $(261.626 \mathrm{~Hz})$. Unit of velocity; $0.5 \mathrm{~mm} / \mathrm{s}$ per division. Driving current $80 \mathrm{~mA}$.
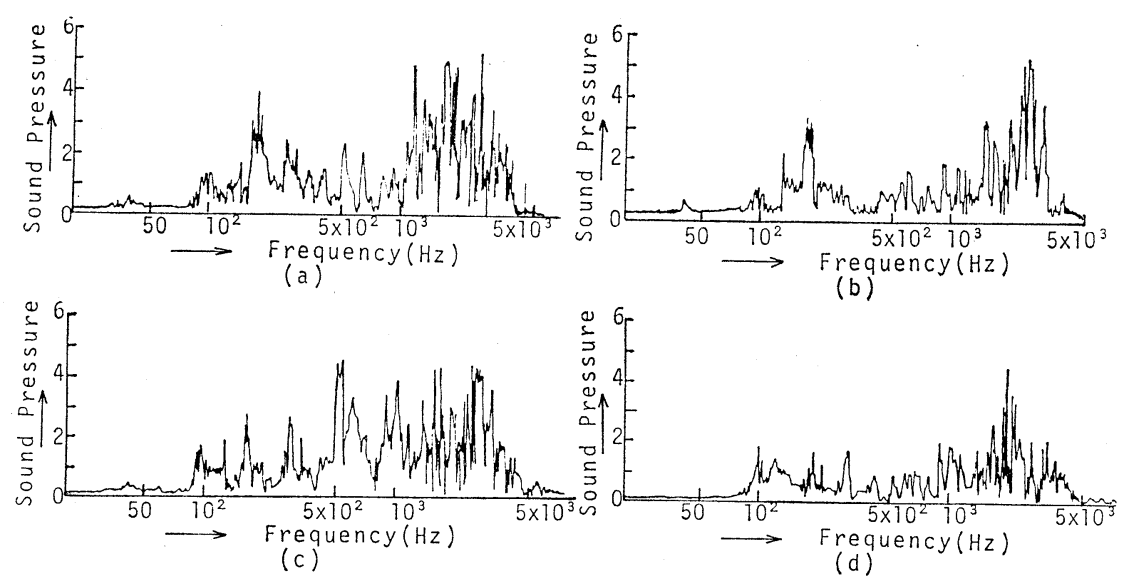

Fig. 12 Frequency characteristic of sound-pressure of a typical soundboard with strings, driven by a constant force. Driving point and frequency:-(a) Key No. 1 (27.5 Hz); (b) Key No. $25(110 \mathrm{~Hz})$; (c) Key No. $27(123.47 \mathrm{~Hz})$; (d) Key No. 40 (261.626 Hz). Sound-pressure in an arbitrary linear scale.

The trend of the frequency characteristic of the sound pressure measured agrees very well with those of the driving-point admittance. The sound pressure characteristics are flatter compared with those of the admittance characteristics; e.g., a region of
$500 \mathrm{~Hz}$ to $1 \mathrm{kHz}$ in the former is generally higher than that in the latter. This is due to the fact that the radiation efficiency of the sound pressure in a higher frequency is better than that in a lower frequency. This suggests that it is sufficient to con- 
sider a frequency region higher than the fundamental frequency for each driving point, and to observe the frequency characteristics of each partial sound. The variation of timbre occurs when the characteristic of each partial harmonic coincides with the variation of the admittance characteristic. Therefore, for some cases it is necessary to obtain the characteristic of each partial harmonic.

For the sound pressure level at a position distant from the soundboard, the directivity of the board must be taken into account. Figure 13 shows sound pressures measured at a point distant from the driving point by $2 \mathrm{~m}$ horizontally, where the driving point is on the centre (height $0.9 \mathrm{~m}$ ) of the soundboard which stands vertically on a floor. The same piano used to obtain Figs. 11 and 12 was used. During the measurements, the piano was set so that its soundboard faces a wall of the room at a distance of $3.8 \mathrm{~m}$. The room wall is covered with rock-wool, glass-wool, and velvet, but this is not a perfectly absorbing condition. Therefore, the measurements were affected by the room conditions, but the results still show a general trend of the phenomenon. The $100 \mathrm{~Hz}$ component is in $[1,1]$ mode, and the $180 \mathrm{~Hz}$ component is in $[3,1]$ mode, and the driving point coincides with their resonance points. The $220 \mathrm{~Hz}$ component is in $[2,2]$ mode, and the driving point coincides with its anti-resonance point. The $[2,2]$ mode has a lower sound pressure than those of $[1,1]$ and $[3,1]$ modes. This is due to the fact that this mode is on the anti-resonance mode, while at

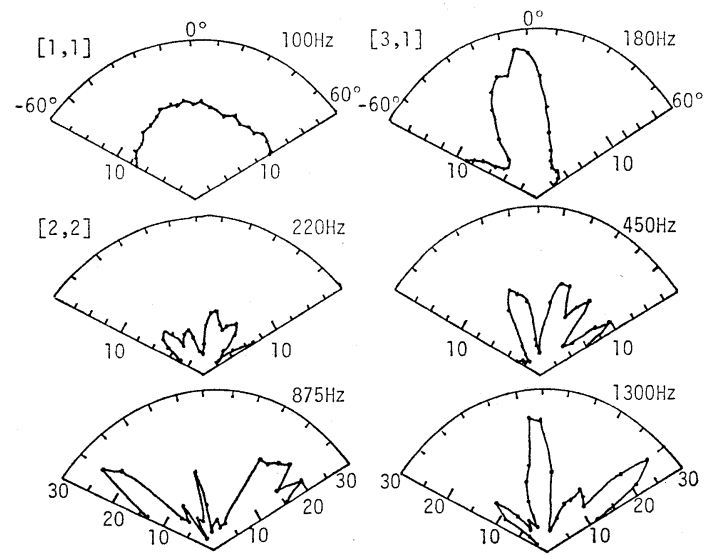

Fig. 13 Directivity of sound radiation from a typical soundboard (measured). Sound pressure in $\mu$ bar. Driving current 300 $\mathrm{mA}$.

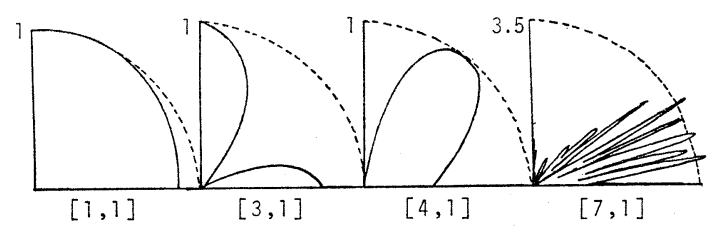

Fig. 14 Directivity of sound radiation from a rectangular plate (theoretical).

the same time each portion viblates anti-symmetrically on both sides of each nodal line. The amplitude of the $450 \mathrm{~Hz}$ component is greater than that of the $220 \mathrm{~Hz}$ component. The efficiency of sound radiation is higher in high-frequency components, and the directivity is sharper. The left-right asymmetry in the radiation patterns seems to be due to the trimming rims. The directivity of sound radiation of the rectangular soundboard is too sharp, and this is reduced by the trimming rims.

Let us consider the theoretical directivities of the rectangular soundboard. Figure 14 shows typical directivities calculated by applying the far-field theory to the simply supported rectangular soundboard. Their $[1,1]$ and $[3,1]$ modes almost agree with the measured patterns. The magnitude of the maximum amplitude is influenced by the drivingpoint characteristic. There is no sound radiation from a theoretical model of the $[2,2]$ mode, because of its anti-symmetry. A small sound radiation occurs in the measured [2,2] mode, since the soundboard is not rectangular and has trimming rims. The $[4,1]$ and $[7,1]$ modes cannot be measured, due to the effects of the trimming rims and the anisotropic plate. However, their patterns are shown in Fig. 14 to help the understanding of the patterns for $450 \mathrm{~Hz}$ and $875 \mathrm{~Hz}$ components.

The discussions in this chapter have clarified the acoustic characteristics of the soundboard, from the point of view of the dring point velocity and the sound pressure, and directivities of far-field.

\section{CONCLUSION}

Complex characteristics of the soundboard of an upright piano have been explained by using various measurements. In a low frequency range, the soundboard vibrates like an isotropic plate due to the effect of its sound ribs. The boundary condition of the soundboard are in an intermediate state between 'simply supported' and 'fixed.' Strings 


\section{NAKAMURA: CHARACTERISTICS OF SOUNDBOARD}

act as a stiffness so that the resonance frequencies of the soundboard increase; and the strings also act as resistances so that the $Q$ of the soundboard decreases. The outline of characteristics of the soundboard can be estimated by measuring the driving-point admittance alone. In a low-frequency range, the driving of the soundboard at its edge makes the amplitude of the fundamental frequency lower, and this forms many higher modes. This structure add to that with series LC resonance system. In a high-frequency range, the vibrations of board parts between sound ribs produces the effective characteristic of the wide range. The trimming rims help to moderate a sharp directivity of the soundboard.

The vibration of the soundboard and its acoustic characteristics are important in understanding the sound generation mechanism of a paino. Some methods described in this paper, such as the measurement of driving-point admittance and the sound-pressure measurement using a constant driving force, are useful in comparing different pianos, and to design new pianos.
This paper is a review of the author's data $^{3,4}$ previously obtained from various measurements on pianos, and is intended to be a part of a systematic study of the piano.

\section{ACKNOWLEDGMENTS}

The author wishes to express his thanks to Dr. Shizuo Ishiguro in England for his advice and help in making this English version.

\section{REFERENCES}

1) I. Nakamura, "A review of acoustical research on pianos," J. Acoust. Soc. Jpn. (J) 35, 447-455 (1979) (in Japanese).

2) P. H. Bilhuber and C. A. Johnson, "The influence of the soundboard on piano tone quality," $\mathrm{J}$. Acoust. Soc. Am. 11, 311-320 (1940).

3) M. Ohtsubaki, I. Nakamura, and A. Kan, "Analysis of the vibrating characteristics of the soundboard," Proc. Spring Meet. Acoust. Soc. Jpn., 135-136 (1967) (in Japanese).

4) I. Nakamura, "Acoustic radiation of a piano," Proc. Autumn Meet. Acoust. Soc. Jpn., 57-58 (1976) (in Japanese). 